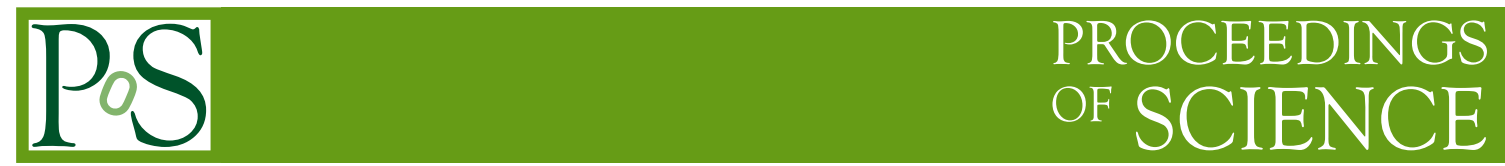

\title{
Heavy flavour phenomenology from lattice QCD
}

\author{
Elvira Gámiz* \\ Department of Physics, University of Illinois, Urbana, IL 61801, USA \\ E-mail: megamizeillinois.edu
}

\begin{abstract}
The focus of this report is the lattice calculation of hadronic parameters relevant to heavy flavour phenomenology. I will review recent results and the current status of studies of the $B$ and $D$ decay constants, semileptonic decay form factors, $B^{0}-\bar{B}^{0}$ mixing, and determinations of the quark masses $m_{c}$ and $m_{b}$. Some studies of heavy flavour observables have used current lattice results to derive Standard Model predictions that disagree with experimental measurements. That is the case of $\sin (2 \beta)$ and $f_{D_{s}}$. This report discusses efforts to resolve the origin of those discrepancies from the lattice side.
\end{abstract}

The XXVI International Symposium on Lattice Field Theory

July 14-192008

Williamsburg, Virginia, USA

${ }^{*}$ Speaker. 


\section{Introduction}

The relevance of lattice calculations of heavy quark quantities is derived not only from their contribution to the extraction of Standard Model (SM) parameters with high precision, but also from their potential to unveil New Physics (NP) effects and put constraints on Beyond the Standard Model (BSM) theories. They are also a good ground to test lattice techniques against well known experimental quantities. This program must be carried out together with and having in mind existing and future experimental measurements. As an example, the new tagged angular analyses of $B_{0} \rightarrow J / \Psi \phi$ by the CDF [1] and DØ[2] collaborations have allowed the extraction of the $B_{s}^{0}$ mixing phase that can be compared against SM predictions that need several inputs from lattice calculations [3]. The disagreement found in that comparison gives the improvement of those lattice inputs primordial importance.

Error analysis is crucial to this effort. Experimental errors of most of the relevant quantities are now at the few percent level. $B^{0}$ mass differences in both the $B_{s}^{0}$ and $B_{d}^{0}$ systems are known with less than $1 \%$ error $[4,5]$. The CLEO-c collaboration has recently improved the determination of both branching fractions of the $D$ and $D_{s}$ leptonic decays, including the measurement of the $D_{s}$ decay to $\tau$ [6]. The experimental error in the extraction of the corresponding decay constants is now 3-4\%. Semileptonic branching ratios of the $D$ meson going to $K$ and $\pi$ allow the extraction $\left|V_{c s}\right|$ and $\left|V_{c d}\right|$ with $2 \%$ and $4 \%$ experimental error respectively [7]. For the extraction of $\left|V_{u b}\right|$ and $\left|V_{c b}\right|$ from $B$ semileptonic decays, experimental errors are $6 \%$ and $1.5 \%$, respectively $[8,9]$. In the study of heavy flavour observables, the main source of uncertainty is thus the error in the theoretical predictions of the non-perturbative inputs. In order to be relevant for phenomenology, according to the experimental numbers listed above, those calculations are needed with accuracy of $5 \%$ or better. For this, realistic sea quarks must be included and all sources of systematic uncertainty must be addressed, with the corresponding errors rigorously estimated. In addition, the validity of the Chiral Perturbation Theory (ChPT) techniques used for extrapolating to the physical point and region of applicability must be checked as discussed at this conference by Karl Jansen [10] and Laurent Lellouch [11]. Now it is possible to perform such calculations and a few examples have already appeared as described at this conference and listed in this review.

The heavy flavour sector is currently very interesting due to the recent claims of possible discrepancies between SM expectations and some flavour observables, for example, the disagreement in the value of the $B_{s}^{0}$ mixing phase reported by the UT collaboration [3] mentioned above. Two other parameters for which disagreement between experiment and SM prediction has been discussed at this conference are the decay constant of the $D_{s}$ meson [12] and the Unitarity Triangle (UT) angle $\sin (2 \beta)$ [14]. All these analyses rely on and are very sensitive to lattice calculations of different quantities: decay constants, the $S U(3)$ breaking ratio of $B^{0}$ mixing parameters $\xi$-defined in Section 4, the form factors needed to extract the Cabibbo-Kobayashi-Maskawa (CKM) matrix matrix elements $\left|V_{c b}\right|$ and $\left|V_{u b}\right|$ from semileptonic decay experimental data,... In the following sections I summarize the advances in those calculations, including updates and new studies, which will be crucial to understand the origin of the discrepancies mentioned above. 


\section{Decay constants}

The lattice determination of pseudoscalar decay constants, together with experimental measurements of pseudoscalar leptonic decay widths, can be used to extract the value of the CKM matrix elements involved in the process via $\Gamma\left(P_{a b} \rightarrow l v\right)=($ known factors $) f_{P}^{2}\left|V_{a b}\right|^{2}$. On the other hand, if the corresponding CKM matrix elements are known from other sources, the experimental measurements can be used to test lattice QCD calculations.

\section{$2.1 f_{D}$ and $f_{D_{s}}$ : The $f_{D_{s}}$ puzzle}

The charm sector was thought to be able to provide valuable tests of lattice QCD techniques, since, in principle, it is not expected to be significantly affected by NP or, at least, it is not expected to be the first place where NP would show up. Important progress has also been made by Bfactories and CLEO-c to reduce the errors in the experimentally measured branching fractions for both $D$ and $D_{s}$. Fixing $V_{c s(d)}=V_{u s(d)}$, one can extract the values of the decay constants from experiment with an error of around $3-4 \%$. When describing charm quarks on the lattice, one must take into account the fact that it falls in a regime which does not correspond to neither the heavy nor the light regimes. Heavy quark effective theories are not able to describe charm quantities with the high precision needed by phenomenology and, on the other hand, relativistic theories need improvements in both actions and operators in order to keep cut-off effects under control and get those needed precisions.

The FNAL/MILC collaboration has presented at this conference the status of the reanalysis of their existing data for the decay constants in the charm and the bottom sectors, and their plans for future runnings [15]. The light quarks in this analysis are simulated with improved staggered quarks, in particular, the Asqtad action [16], and the heavy quarks are simulated following the Fermilab approach [17]. The simulations are performed on MILC configurations with $N_{f}=2+1$ flavours of sea quarks. Three lattice spacings have been analyzed, $a=0.15,0.12,0.09 \mathrm{fm}$, with three or five sea quark masses at each lattice spacing and between nine and twelve light valence quark masses at each sea quark mass choice. The smallest light sea quark mass simulated is $m_{l} \simeq$ $m_{s} / 10$.

An important feature of this analysis is the use of a partially non-perturbative method to renormalize the currents. This reduces the uncertainty in the renormalization procedure compared to the one-loop perturbative approach by a factor of between 2 and 3 (depending on the lattice spacing). Another important feature is the use of Staggered Chiral Perturbation Theory (SChPT) [18] to simultaneously extrapolate the results to the continuum and the physical masses. SChPT allows to remove the dominant light discretization effects, since the expressions explicitly account for the dominant taste-changing violating effects and dependence on the lattice spacing. The downside is that the SChPT expressions are rather complicated and depend on a large number of parameters that must be fixed using other simulations or as an output of the extrapolation fits. These two approaches are common to most of all FNAL/MILC calculations mentioned in this paper except for the $B^{0}-\bar{B}^{0}$ study for which the applicability of the partially non-perturbative renormalization is still under investigation. In those analyses at least NLO SChPT expressions are considered and, in most of the cases, analytic NNLO terms are also included in the fits. 

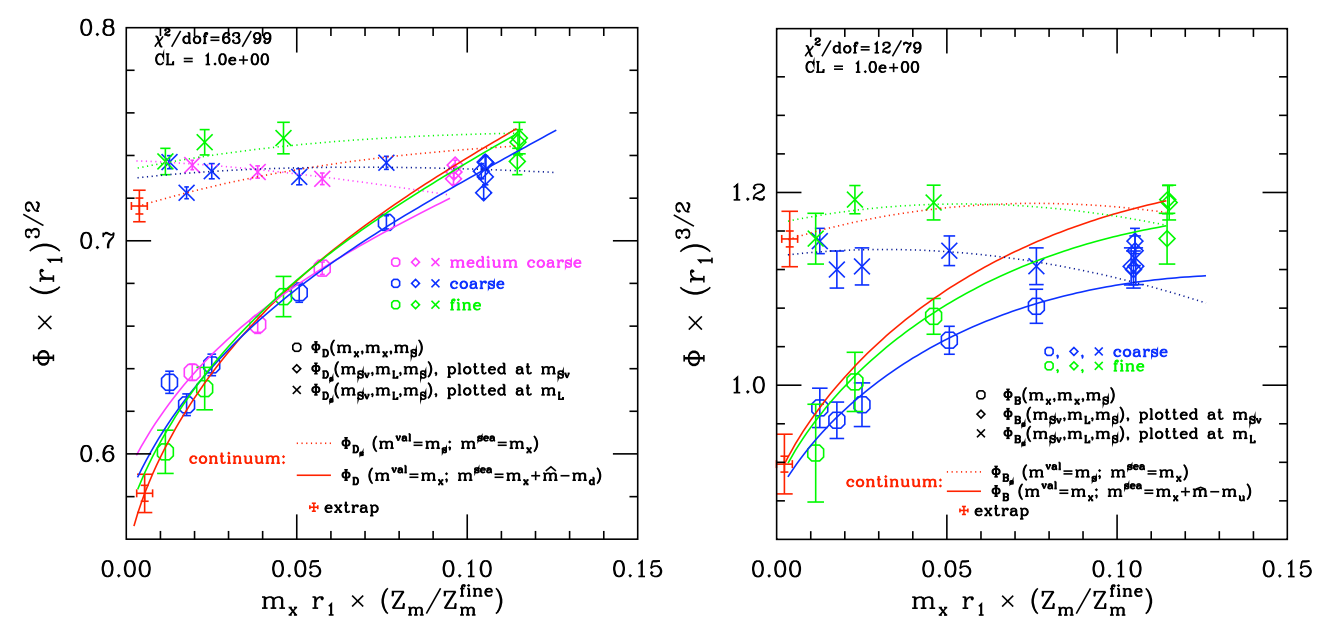

Figure 1: $\Phi=f_{D_{q}} \sqrt{M_{D_{q}}}$ and $\Phi=f_{B_{q}} \sqrt{M_{B_{q}}}$ in $r_{1}$ units. Hexagons correspond to the $D / B$ parameters plotted as a function of the light valence quark mass with the sea masses fixed to the physical values. Crosses correspond to $D_{s} / B_{s}$ parameters plotted as a function of the sea light quark mass. Figure taken from [15].

The extrapolated values of $f_{D}$ and $f_{D_{s}}$ are extracted from the same fit, whose results are shown in Figure 1. The dependence on the light sea quark mass is very mild as can be seen in that figure. The light quark masses are light enough so the lattice data show the correct behaviour dictated by the logarithms in the chiral expressions. The results obtained for the two decay constants and the ratio are

$$
f_{D}=207(11) \mathrm{MeV} \quad f_{D_{s}}=249(11) \mathrm{MeV} \quad f_{D_{s}} / f_{D}=1.200(27) .
$$

The errors in (2.1) are dominated by the tuning of $m_{c}$ and discretization errors. They are planning to improve this calculation by performing simulations at $a=0.06 \mathrm{fm}$, as well as on the extra configurations generated on the existing ensembles. In addition, they are currently retunning the charm quark mass. These improvements can be applied to all the FNAL/MILC analyses discussed in this paper.

The FNAL/MILC results can be compared with the HPQCD calculation in [19] that employs the same $N_{f}=2+1$ MILC gauge configurations. The main difference between the two calculations is the treatment of the valence quarks, described by the HISQ action [20] in the HPQCD analysis. Other differences are the fact that HPQCD has no need of renormalization (they use PCAC relations to extract the decay constants) and instead of using SChPT to perform the extrapolation to the continuum and the physical masses they perform a Bayesian analysis including continuum NLO ChPT plus different $O\left(a^{2}\right)$ functional forms, and second and third order polynomial in the masses. In addition, the HPQCD collaboration only studied full QCD points. Their results

$$
f_{D}=(208 \pm 4) \mathrm{MeV} \quad f_{D_{s}}=(241 \pm 3) \mathrm{MeV} \quad f_{D_{s}} / f_{D}=1.162(9)
$$

agree very well with the FNAL/MILC ones, although with smaller errors due mainly to the fact that the valence quark action for the $b$ quark is more improved.

Another interesting on-going calculation, albeit less complete, of decay constants in the charm sector is the one by the ETM Collaboration whose preliminary results have been presented at this 


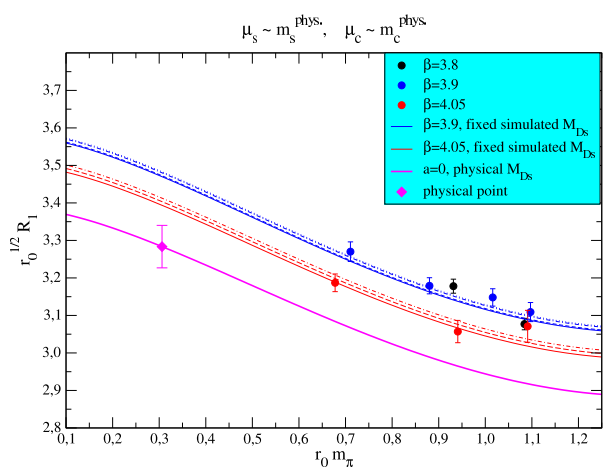

Figure 2: Chiral extrapolation for the ratio $R_{1}$ defined in the text as a function of $r_{0} m_{\pi}$ for the three lattice spacings analyzed and the corresponding continuum extrapolation. Figure is taken from [21].

conference [21]. They employ a twisted mass (tmQCD) formalism with $N_{f}=2$ light sea quarks. The hadronic quantities like meson masses and decay constants are automatically $O(a)$ improved using this formalism at maximal twist [22]. In addition, quark masses are multiplicatively renormalized (and the renormalization factor can be determined non-perturbatively) and decay constants do not require to be renormalized. They are extracted from PCAC relations. In this study full QCD points for light quark masses between $m_{s} / 5$ and $m_{s} / 2$ are analyzed for three lattice spacings, $a=0.1,0.0855,0.0667 \mathrm{fm}$. Simulations are performed for several values of the charm quark mass around the physical one. A good way of doing the chiral extrapolation milder is by considering ratios of decay constants. The ETM collaboration use the ratios $R_{1}=f_{D_{s}} / f_{K}$ and $R_{2}=\left[f_{D_{s}} \sqrt{M_{D_{s}}} / f_{K}\right] /\left[f_{D} \sqrt{M_{D}} / f_{\pi}\right]$, and their own results for $f_{K}$ and $f_{K} / f_{\pi}$ to extract the decay constants in the charm sector. The chiral extrapolation is performed together with the extrapolation to the continuum through a simultaneous fit analogous to the ones employed by the HPQCD collaboration and described above. Together with the continuum NLO Heavy Meson ChPT expressions (HMChPT $)^{1}$ explicit dependence in $a^{2}$ is also included in the functional form. The results from this simultaneous fit for $R_{1}$ are shown in Figure 2. The preliminary results presented in [21] are

$$
f_{D}=(197 \pm 7 \pm 12) \mathrm{MeV} \quad f_{D_{s}}=(244 \pm 4 \pm 11) \mathrm{MeV} \quad f_{D_{s}} / f_{D}=(1.24 \pm 0.04 \pm 0.02),
$$

where the first error is statistical and the second systematic. As can be seen in Figure 2, there is a significant gap between the results for the smallest lattice spacing and the continuum extrapolated numbers, which translates into a sizable uncertainty associated with the continuum extrapolation. This analysis needs a better control over discretization errors. The collaboration is planning to perform simulations at a smaller lattice spacing $a \simeq 0.06 \mathrm{fm}$ to address that limitation. The results in (2.3) agree with the ones quoted by the collaborations simulating $N_{f}=2+1$ sea quarks. However, the effects of including a third sea quarks are missing and the systematic analysis does not include the corresponding error.

If we take the most recent lattice results for $f_{D}$ and $f_{D_{s}}$ and compare then with experiment -as has been done in Figure 3-, it can be seen that the agreement for $f_{D}$ is very good, but for $f_{D_{s}}$ there

\footnotetext{
${ }^{1}$ The authors in [21] claim that the strange quark mass could be too heavy to be described by ChPT, so they use SU(2) HMChPT expressions (obtained from the SU(3) ones by performing and expansion in $m_{l} / m_{s}$ ) and use SU(3) expressions to estimate the corresponding systematic error.
} 
is a clear tendency for lattice calculations to give smaller values than experiment. In particular, the HPQCD result, which is the most accurate one, is more than $3 \sigma$ away from experiment. The
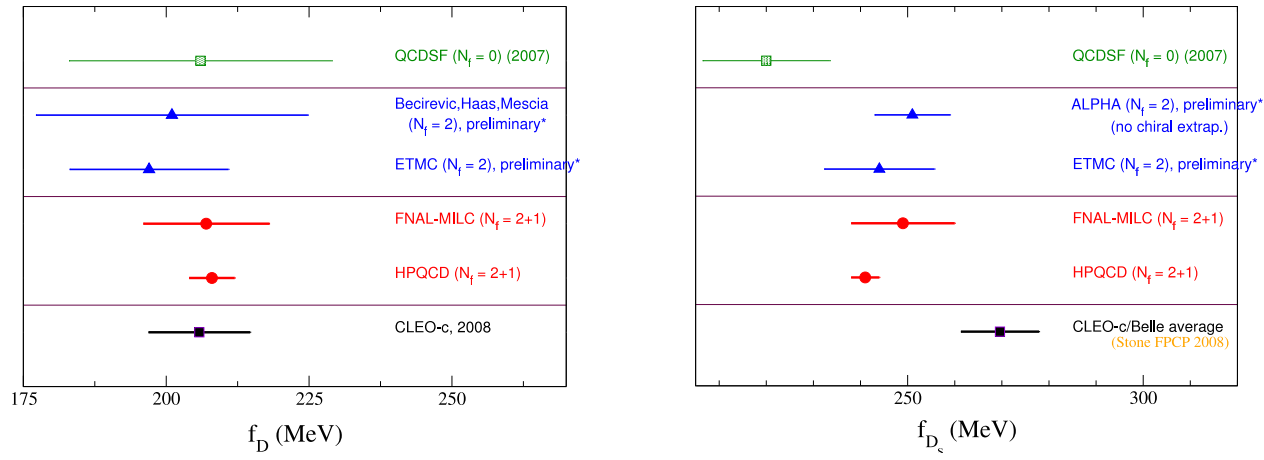

Figure 3: Values of $f_{D}$ and $f_{D_{s}}$ from experiment [23, 24] and recent lattice calculations.

fact that the rest of quantities calculated by the HPQCD calculation with the same actions, configurations, input parameters, etc $\left(f_{D}, f_{K}, f_{\pi}, m_{D}, m_{D_{s}}, \frac{2 m_{D_{s}}-m_{\eta_{c}}}{2 m_{D}-m_{\eta_{c}}}, \ldots\right)$ agree with experiment at the $2 \%$ level, strongly supports the reliability of their results for $f_{D_{s}}$. In any case, it would be good to have an independent $N_{f}=2+1$ lattice calculation with comparable errors of this quantity. On the experimental side, it would be also interesting that some issues like the use of unitarity to fix the value of $V_{c s}$ or the more careful treatment of radiative corrections were addressed. On the other hand, it is possible that this discrepancy is indicating the presence of NP effects as discussed at this conference [12].

The ALPHA collaboration has also presented preliminary results for $f_{D_{s}}$ as well as the charm quark mass at this conference [13]. Those are simulations with $N_{f}=2$ Wilson fermions which seems to indicate that cutoff effects are under control due to the small lattice spacings used (finest lattice spacing is $a=0.04 \mathrm{fm}$ ).

\section{$2.2 f_{B}$ and $f_{B_{s}}$}

The decay constants for leptonic $B$ and $B_{s}$ mesons are also parameters of phenomenological relevance. Lattice results for the decay constants in the $B$-meson sector are needed more than in the $D$-meson sector since the corresponding CKM matrix elements. The $B$ leptonic decays themselves would also be a sensitive probe of effects from charged Higgs bosons. The $B$ decay constants are also used in the SM predictions for processes very sensitive to beyond SM effects, such as $B_{s} \rightarrow \mu^{+} \mu^{-}$.

One of the advantages of the Fermilab action is that it can be efficiently used to describe both charm and bottom quarks. This has allowed the FNAL/MILC collaboration to use the same setup as the one described in section (2.1) to calculate $f_{B}, f_{B_{s}}$, and the ratio of both decay constants. The results from the simultaneous chiral and continuum extrapolations for $f_{B}$ and $f_{B_{s}}$ are shown in Figure 1. After the extrapolations the numbers obtained are $f_{B}=(195 \pm 11) \mathrm{MeV}, f_{B_{s}}=(243 \pm$ $11) \mathrm{MeV}$ and $f_{B_{s}} / f_{B}=1.25 \pm 0.04$. Statistics is in this case a more important source of error than for $f_{D}, f_{D_{s}}$, while in the $B$ sector results are less sensitive to the tuning of the $b$ quark mass.

These results agree with the $N_{f}=2+1$ calculation of the HPQCD collaboration [25] using also the MILC configurations but with $b$ quarks simulated with the NRQCD action in [26]: $f_{B}=$ 
$(216 \pm 22) \mathrm{MeV}, f_{B_{s}}=(260 \pm 26) \mathrm{MeV}$ and $f_{B_{s}} / f_{B}=1.20 \pm 0.03$. The errors in HPQCD numbers are larger by around a factor of two mainly due to the uncertainty associated with the one-loop perturbative renormalization used for the matching of the corresponding currents.

There have been two recent quenched calculations of $f_{B_{s}}$ using $O(a)$ improved Wilson fermions but following different approaches, both going beyond the static approximation. The authors in [27] performed simulations using a relativistic description with heavy masses around the charm quark mass together with simulations in the static approximation. They interpolated the results to the physical point using the expression $r_{0}^{3 / 2} \frac{F_{P S} \sqrt{m_{P S}}}{C_{P S}\left(M / \Lambda_{\overline{M S}}\right)}=A\left(1+\frac{B}{r_{0} m_{P S}}\right)$. The slope leads to a $1 / m_{P S}^{2}$ correction of about $10 \%$ at the physical point. The number obtained is $f_{B_{s}}=191(6)$, where all errors except quenching are considered. The other quenched analysis, described in [28], is based on the use of the Step Scaling Method (SSM) [29] and the Heavy Quark Effective Theory (HQET). The authors calculate the SS functions for several heavy masses around the charm quark mass and in the static approximation. They then extract the final by performing an interpolation in $1 / \mathrm{m}$ to the physical $B_{s}^{0}$ mass. As expected, SS functions were found to be very weakly dependent on the mass. The result obtained is $f_{B_{s}}=193(7)$. In this analysis, as well as the one in [27], the inclusion of the static point improves noticeably the control over the heavy quark mass dependence. Both numbers in [27] and [28] are in very good agreement, which gives confidence to the systematic error analyses. It is clear from the results obtained in these two calculations and those from the $N_{f}=2+1$ calculations mentioned above that the effect of the quenched approximation is quite noticeable in $f_{B_{s}}$. However, the quenched results already show that the two methods are promising to get accurate values of $f_{B_{s}}$. It would be also interesting to see whether these two results agree with a pure HQET calculation including $1 / M$ corrections.

Several collaborations working on static-light studies have presented preliminary results at this conference. The RBC/UKQCD collaboration has completed the one-loop renormalization calculations needed for its $f_{B}$ and $B^{0}-\bar{B}^{0}$ analyses with $N_{f}=2+1$ sea quarks and domain wall light quarks [30]. The ETMC collaboration is using twisted mass fermions with $N_{f}=2$ together with static bottom quarks. They have presented first results for the spectrum of $B_{s}$-mesons [31]. And beyond the static limit, the HPQCD collaboration reported also at this conference preliminary results for the calculation of $B_{c}$ and $B_{s}$ meson masses with NRQCD and HISQ fermions [32].

\section{Semileptonic decays}

Semileptonic decays of heavy-light mesons are currently used to extract the CKM matrix elements $\left|V_{c b}\right|,\left|V_{u b}\right|,\left|V_{c d}\right|$ and $\left|V_{c s}\right|$. The theory input needed to get those parameters from experimentally measured semileptonic widths are the form factors in terms of which the hadronic matrix elements involved on those decays are parametrized. For example, for the decay $D \rightarrow K l v$, the differential decay rate is given by $\frac{d \Gamma}{d q^{2}}=($ knownfactors $)\left|V_{c s}\right|^{2} f_{+}^{2}\left(q^{2}\right)$, where $f_{+}\left(q^{2}\right)$ is the vector form factor. This vector form factor can be extracted from the matrix element of the vector current $\left\langle K\left|V^{\mu}\right| D\right\rangle=f_{+}\left(q^{2}\right)\left(p_{D}+p_{K}-\Delta\right)^{\mu}+f_{0}\left(q^{2}\right) \Delta^{\mu}$, with $\Delta^{\mu}=\left(m_{D}^{2}-m_{K}^{2}\right) q^{\mu} / q^{2}$ and $q=p_{D}-p_{K}$. Analogously, the processes with a vector meson in the final state are described by four form factors.

There are several lattice techniques that are considerably contributing, or will do in the near future, to the improvement of the calculations of semileptonic form factors. An important reduction of the errors is got by using double ratios methods [33], whose goal is the cancellation of statistical 
and systematic errors as well as chiral corrections between denominator and numerator. The use of ratios often also yields a milder chiral extrapolation. Another important ingredient to be considered when calculating form factors is the parametrization used to describe the $q^{2}$ dependence. Traditional lattice QCD methods are able to calculate form factors in an accurate way only at high values of $q^{2}$, while experimental values are more precise for low $q^{2}$. One thus needs a description of the $q^{2}$ dependence to connect both set of results. The optimal choice is a model independent parametrization, as discussed later in section 3.2. On the other hand, with twisted boundary conditions [34] the simulations can be performed at smaller recoil momenta than with periodic boundary conditions, which allows a better resolution of the small recoil region.

Another approach to deal with the large discretization errors at small values of $q^{2}$ is using a fermion formulation that address directly the issue. This is the case of moving nonrelativistic QCD (mNRQCD), with which discretization errors can be kept under control for small values of $q^{2}$. Preliminary results for the first calculation of form factors using $\mathrm{mNRQCD}$ have been presented at this conference [35]. The statistical errors are still large, but the authors will improve them by using random wall sources.

\subsection{Exclusive $B \rightarrow D^{*}(D) l v$ : determination of $\left|V_{c b}\right|$}

The reduction in the error of $\hat{B}_{K}$ under $10 \%$ [11] has made the error in the determination of the CKM matrix element $\left|V_{c b}\right|$ to become a dominant source of uncertainty in the analysis of $\varepsilon_{K}$, which measures indirect CP violation in the Kaon system. This CKM matrix element is also needed in the analysis of some rare kaon decays, for example, $K \rightarrow \pi v \bar{v}$.

There are two processes that have been used to extract the value of $\left|V_{c b}\right|$ using lattice techniques, $B \rightarrow D l v$ and $B \rightarrow D^{*} l v$. In general, the analysis of these processes depend on a combination of four form factors which are functions of $\omega$ (the scalar product of the velocities of the meson in the initial and the final state), but at zero recoil $(\omega=1)$ only one form factor, $h_{A_{1}}$, is needed. The experimental results for $B \rightarrow D^{*} l v$ at zero recoil have smaller errors than those for $B \rightarrow D l v$, so it yields a more precise determination of $\left|V_{c b}\right|$.

The FNAL/MILC collaboration presented last year at this conference [36] a preliminary result for its calculation of $h_{A_{1}}$. This analysis has recently been completed [37]. They perform a $N_{f}=$ $2+1$ calculation with Asqtad staggered light quarks and heavy quarks described with the Fermilab formalism. The analysis introduces a new double ratio method which gives the form factor at zero recoil directly. There is also a reduction of the computational cost from previous FNAL/MILC analyses [38] since the new method does not require simulations at several heavy quark masses. The relation between the double ratio and the form factor,

$$
\left|\mathscr{F}_{B \rightarrow D^{*}}(1)\right|^{2}=\frac{\left\langle D^{*}\left|\bar{c} \gamma_{j} \gamma_{5} b\right| \bar{B}\right\rangle\left\langle\bar{B}\left|\bar{b} \gamma_{j} \gamma_{5} c\right| D^{*}\right\rangle}{\left\langle D^{*}\left|\bar{c} \gamma_{4} c\right| D^{*}\right\rangle\left\langle\bar{B}\left|\bar{b} \gamma_{4} b\right| \bar{B}\right\rangle}
$$

is exact to all orders in the heavy-quark expansion in the continuum. Statistical errors in the numerator and denominator are highly correlated and largely cancel. Most of the renormalization also cancels, yielding a small uncertainty for the perturbative matching. Figure 4 shows the mass and lattice spacing dependence of the data. In both cases the extrapolation needed to go to the physical/continuum limit is very mild. The final result obtained for the form factor after chiral and continuum extrapolation is $h_{A_{1}}(1)=0.921 \pm 0.013 \pm 0.021$ [37], where the first error is statistical 

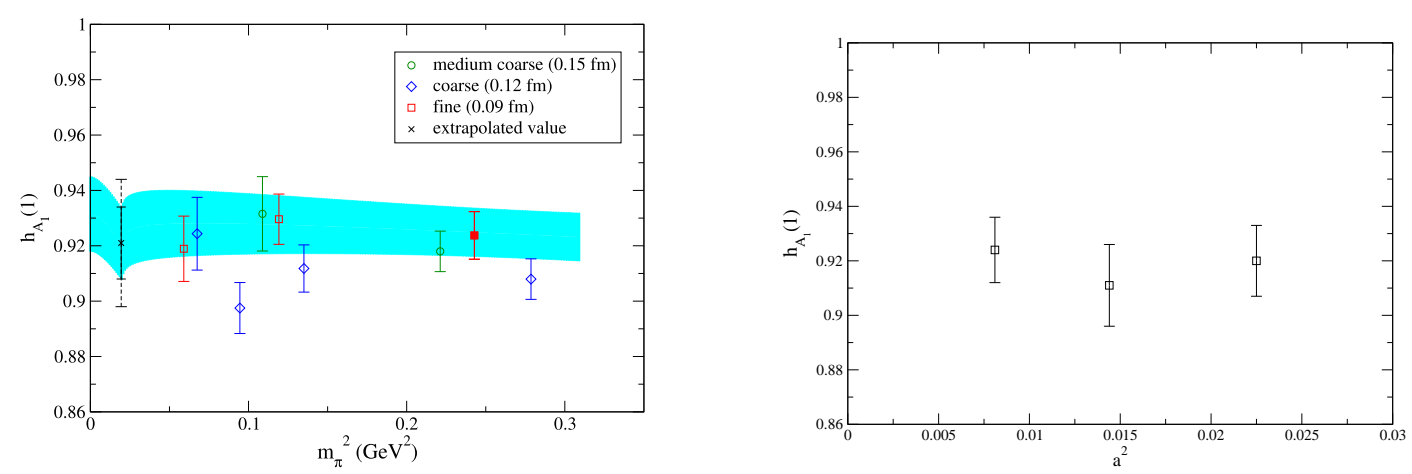

Figure 4: The figure in the left shows the full QCD points for the form factor $h_{A_{1}}(1)$ versus $m_{\pi}^{2}$ on the three lattice spacings. The curve is already extrapolated to the physical strange sea quark mass. The cross is the extrapolated value with statistical (solid line) and systematic (dashed line) errors. The figure in the right shows $h_{A_{1}}(1)$ at physical quark masses versus $a^{2}\left(\mathrm{fm}^{2}\right)$. Figures taken from [37].
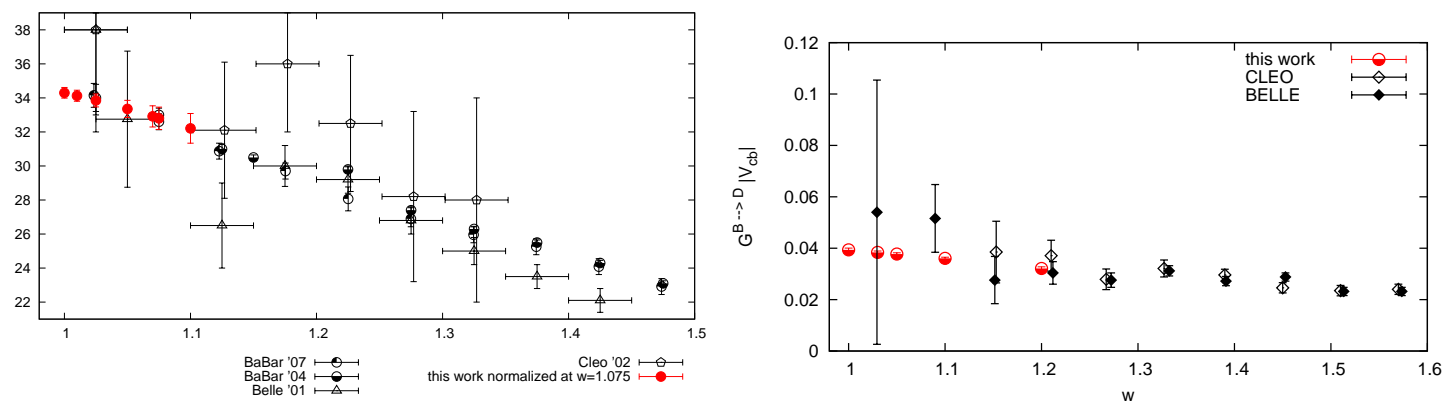

Figure 5: Comparison of experimental data and quenched lattice data for $B \rightarrow D^{*}$ form factors [39] and $B \rightarrow D$ form factor [40] respectively. The value of $\left|V_{c b}\right|$ in this plot lattice is extracted from matching experimental and lattice data at $\omega=1.075$ for $B \rightarrow D^{*}$ and $\omega=1.2$ for $B \rightarrow D$. Figures from [39] and [40].

and the second one includes all sources of systematic errors and it is dominated by heavy-quark discretization errors. The CKM matrix element $\left|V_{c b}\right|$ extracted from this value of the form factor and the experimental averages in [9] is $\left|V_{c b}\right|=\left(38.8 \pm 0.6_{\text {exp }} \pm 1.0_{\text {theo }}\right) \times 10^{-3}$. This value differs by $2 \sigma$ from the one extracted from inclusive decays [5], $\left|V_{c b}\right|=(41.7 \pm 0.7) \times 10^{-3}$. In this FNAL/MILC calculation all the sources of error are under control, none of them being larger than $1.5 \%$ [37], giving to a very clean extraction of $\left|V_{c b}\right|$.

The recent quenched study in [39] calculates the combination of form factors needed to describe $B \rightarrow D^{*} l v$ for values of $\omega \geq 1, F^{B \rightarrow D^{*}}(\omega)$, using the Step Scaling method. These values of $\omega$ can be reached thanks to the use of twisted flavour boundary conditions. Although it is a quenched calculation, it is useful to check the rest of the systematics entering in the analysis and to discuss some technical issues, having in mind the future inclusion of vacuum polarization effects. The results obtained for the product $F^{B \rightarrow D^{*}}\left|V_{c b}\right|$ together with different experimental measurements are shown in the left hand side of Figure 5. There is a very good overlap between lattice and experimental data for the region of $\omega$ studied, but in this process it is not so important to go to larger 
values of $\omega$ since for $\omega=1$ experimental data are already good. That is however crucial for the decay $B \rightarrow D l v$ where, as can be seen in the right hand side of Figure 5, experimental data at zero recoil are very noisy. That figure corresponds to the quenched analysis of $B \rightarrow D l v$ in [40], which follows the same methodology than the one for $B \rightarrow D^{*} l v$ in [39]. An interesting feature of the analysis in [40] is that the authors determine the ratio $\Delta^{D \rightarrow B}(\omega)$, which parametrizes the difference between $B \rightarrow D e(\mu) v_{e}\left(v_{\mu}\right)$ and $B \rightarrow D \tau v_{\tau}$. This ratio could be extracted from the experimental measurement of $d \Gamma\left(B \rightarrow D \tau \nu_{\tau}\right) / d \Gamma\left(B \rightarrow D e(\mu) v_{e}\left(v_{\mu}\right)\right)$ and it is independent of CKM inputs, so it potentially constitutes a good way to check lattice techniques. The calculation of $\Delta^{D \rightarrow B}(\omega)$ is also relevant because the ratio of partially integrated rates $\operatorname{Br}\left(B \rightarrow D \tau \nu_{\tau}\right) / B r\left(B \rightarrow D e v_{e}\right)$ has been claimed to be a good place to look for charged Higgs contributions to low energy observables [41]. Having results for both channels makes also possible to perform lepton-flavour universality checks on the extraction of $\left|V_{c b}\right|$.

\subsection{Exclusive $B \rightarrow \pi l v$ : determination of $\left|V_{u b}\right|$}

The decay $B \rightarrow \pi l v$ provides a determination of $\left|V_{u b}\right|$ that is competitive with inclusive $b \rightarrow u$ decays. The main problem in studying the exclusive channel with lattice techniques is the poor overlap in the momentum transfer, $q^{2}$, between experimental and lattice data, which inflates the final error. A solution to this problem is using a model independent parametrization of the shape of the form factor. This allows the direct comparison of experimental and lattice data even with a poor $q^{2}$ overlap.

This is the approach that is being followed by the FNAL/MILC collaboration [42, 43]. The authors in $[42,43]$ used the so called $z$-expansion, which is a model independent parametrization based only on unitarity and analyticity [44]. Analyticity, unitarity and heavy quark symmetry can be combined together with experimental data to determine the shape of the form factors. Lattice simulations must then only provide a normalization that can be extracted from the region where lattice data are most precise. The FNAL/MILC collaboration is calculating the form factors for three different values of the lattice spacing and for full QCD points. The results are extrapolated to the continuum and physical masses using SChPT. In the case of the form factor $f_{\perp}$, which dominates the value of $f_{+}$defined above, a NLO description is enough to have good fits since this form factor is dominated by the $B^{*}$ pole. Results from the extrapolation are shown in Figure 6 . The extrapolated lattice data are then fitted together with the experimental BaBar measurements [8] using the $z$-expansion mentioned before and leaving $\left|V_{u b}\right|$ as one of the three parameters to be determined by the fit. The output of a preliminary analysis, which is plotted in Figure 6, is $\left|V_{u b}\right|=(2.94 \pm 0.35) \times 10^{-3}$ [42]. This value, although still preliminary, is consistent with the global fits of the CKM matrix [45] but it is $2 \sigma$ away from the inclusive values [9]. In this analysis statistics is one of the first things that need to be improve, since it is one of the uncertainties dominating the $12 \%$ error in $\left|V_{u b}\right|$. The other dominant error is the one associated with the chiral and continuum extrapolation, which is also enhanced by the lack of good statistics.

The authors in [46] have recently proposed a new model independent parametrization, which satisfies unitarity, analyticity and perturbative scaling. As an illustration of their method, they fit their parametrization of the form factor $f_{+}$for $B \rightarrow \pi l v$ to experimental, existing lattice and light cone sum rules results. The form factors obtained yield to a value of $\left|V_{u b}\right|$ that agrees with the one by the FNAL/MILC collaboration. 

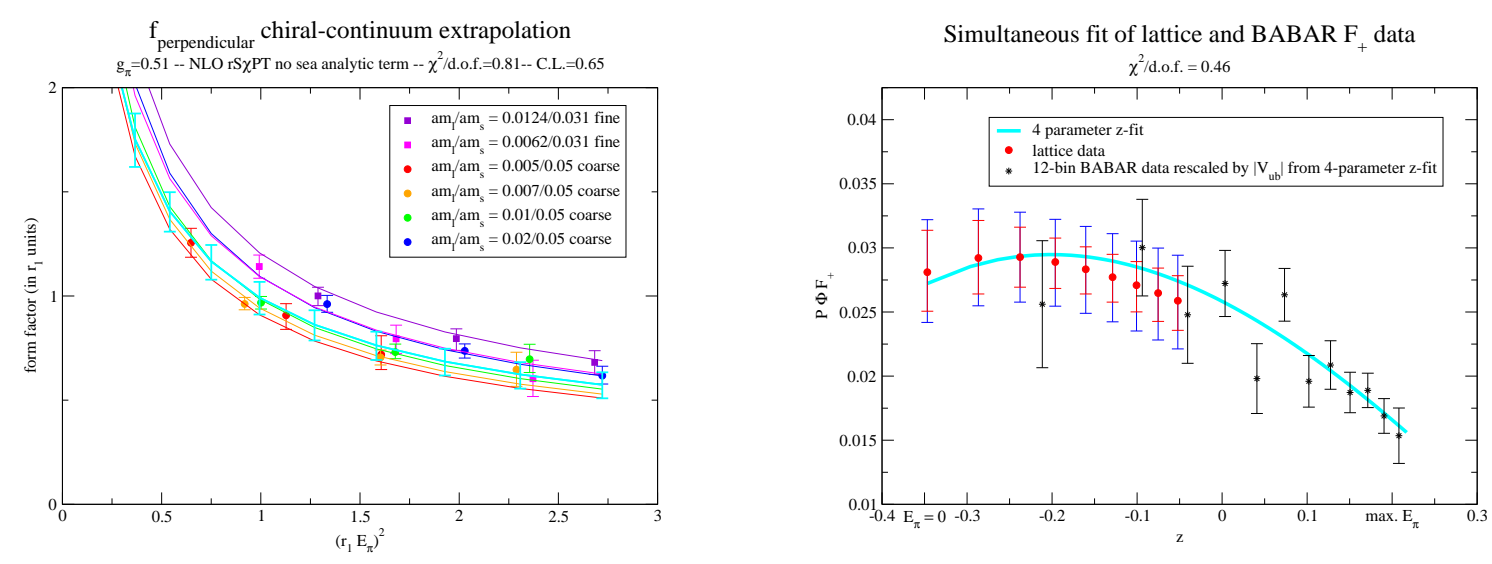

Figure 6: The two figures are taken from [42]. The one in the right hand side shows the chiral and continuum extrapolation for $f_{\perp}$ which dominates the value of $f_{+}$. The second figure shows the combined z-fit of lattice and BaBar data from which the preliminary value of $\left|V_{u b}\right|$ in [42] is obtained.

The QCDSF collaboration is performing a quenched systematic study of several semileptonic decays [47]. They use simulations with $O(a)$ improved Wilson fermions at a single lattice spacing, $a=0.04 \mathrm{fm}$. The small value of $a$ allows them to simulate at the physical charm quark mass and bottom masses very close to the physical ones. But the light masses are quite heavy, $m_{\pi}^{\min }=526 \mathrm{MeV}$. The authors use the Becirevic-Kaidalov (BK) parametrization [48] to describe the dependence on the momentum transfer, introducing a model dependency in their analysis. The preliminary numbers for the form factors are $f_{+}^{B \rightarrow \pi}(0)=0.232(23), f_{+}^{B \rightarrow K}(0)=0.29(3)$, $f_{+}^{D \rightarrow \pi}(0)=0.668(38), f_{+}^{D \rightarrow K}(0)=0.733(38)$ and $f_{+}^{D_{s} \rightarrow K}(0)=0.598(20)$; where errors include both statistic and systematic uncertainties other than quenching and the one associated to the model use in the $q^{2}$ extrapolation.

The ratio of form factors $f_{+}^{B \rightarrow K}(0) / f_{+}^{B \rightarrow \pi}(0)$ is needed as an input in the prediction of the SM correlation between $S_{\pi^{0} K_{S}} \equiv(\sin 2 \beta)_{\pi^{0} K_{S}}$ and $A_{\pi^{0} K_{S}}$, the mixing-induced and direct CP asymmetries of $B^{0} \rightarrow \pi^{0} K_{S}$ [49]. The current SM prediction, which assumes the ratio to be equal to 1 , is not in agreement with experiment, so it would be very important to study this channel to have an accurate determination of this ratio including sea quark effects in a realistic way.

\section{3 $D \rightarrow \pi(K) l v$ : determination of $\left|V_{c d(c s)}\right|$}

Good quantities for testing lattice QCD are the ratios of semileptonic and leptonic decay widths

$$
\frac{1}{\Gamma\left(D^{+} \rightarrow l v\right)} \frac{d \Gamma(D \rightarrow \pi l v)\left(q^{2}\right)}{d q^{2}} \quad \frac{1}{\Gamma\left(D_{s} \rightarrow l v\right)} \frac{d \Gamma(D \rightarrow K l v)\left(q^{2}\right)}{d q^{2}}
$$

Or, equivalently, ratios of semileptonic form factors and decay constants. An advantage of using these ratios is that the chiral extrapolation to the physical pion mass is milder than for denominator or numerator alone. 


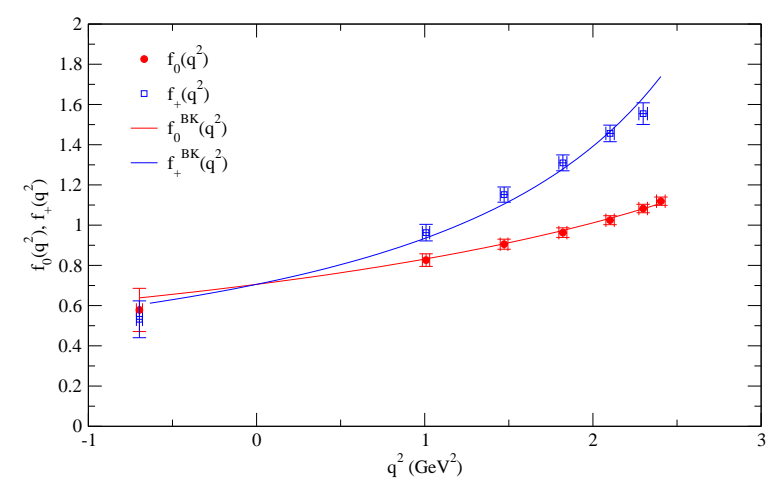

Figure 7: Data points for the form factors $f_{+}^{D \rightarrow \pi l v}\left(q^{2}\right)$ and $f_{0}^{D \rightarrow \pi l v}\left(q^{2}\right)$ for different values of $q^{2}$ together with the corresponding BK parametrizations $f_{+(0)}^{B K}\left(q^{2}\right)$. Figure courtesy of S. Simula.

On the other hand, these $D$ semileptonic decays can be used to extract the CKM matrix elements $\left|V_{c s}\right|$ and $\left|V_{c d}\right|$. In fact, the experimental measurement of the branching ratio $\operatorname{Br}(\mathrm{D} \rightarrow \mathrm{Kev})$ and the lattice calculation of the corresponding form factors by the FNAL/MILC collaboration [50] are the inputs of the current best determination of $\left|V_{c s}\right|=1.015 \pm 0.015 \pm 0.106$. The semileptonic decay $D \rightarrow \pi e v$ has also the potential to provide the most accurate result for $\left|V_{c d}\right|$ if errors in the lattice calculation of the form factors are reduced to the $10 \%$ level or less.

The ETMC collaboration is calculating the form factors for $D \rightarrow \pi(K) l v$ using $N_{f}=2$ twisted mass QCD at maximal twist. The main new techniques applied in this calculation are the allto-all propagators obtained with a stochastic method and twisted boundary conditions. The main limitations of the preliminary results in [51] are the fact that the simulations are performed at a single lattice spacing and the use of a BK parametrization to describe the $q^{2}$ dependence. The preliminary results obtained for $D \rightarrow \pi l v$ as a function of $q^{2}$ can be seen in Figure 7. The physical pion masses are achieved by an extrapolation of the data for the ratio of the form factor $f_{+}\left(q^{2}\right)$ and the decay constant $f_{D}$ with $0.3 \leq m_{\pi}(\mathrm{GeV}) \leq 0.6$. They reached the physical $D$ mass by an interpolation of results at four different heavy quark masses around $m_{c}$.

Becirevic, Haas and Mescia also presented preliminary results of their calculation of the form factors for $D \rightarrow \pi l v$ with $N_{f}=2$ flavours of sea quarks, employing the configurations by the QCDSF collaboration in [52]. They use $O(a)$ improved Wilson fermions at a single lattice spacing $a \simeq 0.08 \mathrm{fm}$, pion masses $m_{\pi}=770,585,380 \mathrm{MeV}$ and a fixed value of the charm valence quark mass around the physical one. The method employed is the double ratio strategy 1 described in [53], where the $D$ meson is at rest and the momentum is injected to the pion. Twisted boundary conditions are also used in this work. The preliminary results obtained for $f_{+}$are collected in Figure 8 for the different light quark masses simulated. In this Figure, one can appreciate a qualitative change in the shape of the the form factor, that also occurs for $f_{0}$, when $m_{\pi}$ goes to the physical value. That corresponds to the better resolution of the polar behaviour due to $m_{D^{*}}$ when the data are moving closer to the physical kinematic region and point out the importance of having light masses small enough. The chiral extrapolation in this work is also done for the ratio of the semileptonic form factor and the decay constant. The authors performed both a extrapolation using HMChPT and a linear extrapolation, since they didn't observe any sensitivity of their data to the logarithms. 


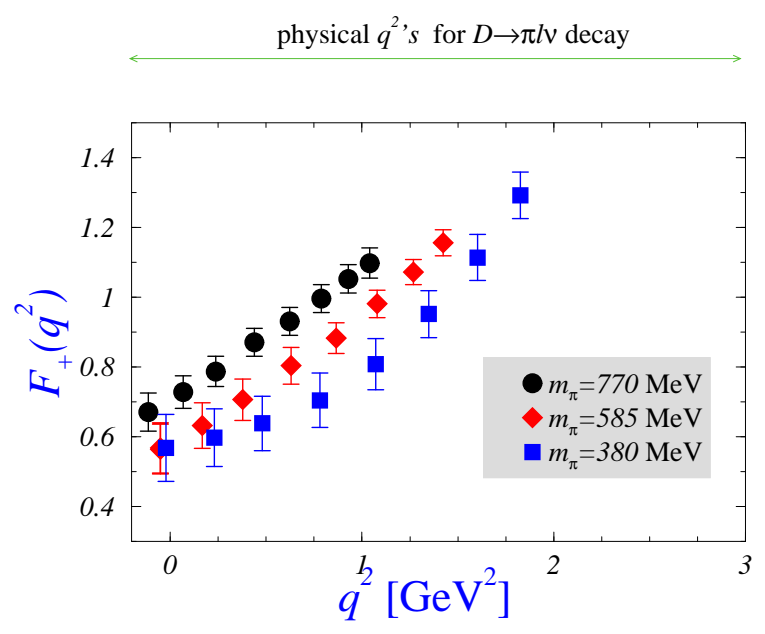

Figure 8: Results for the form factor $f_{+}^{D \rightarrow \pi l v}\left(q^{2}\right)$ from simulations using three different values of the light quark masses. Figure courtesy of B. Haas.

\begin{tabular}{cc}
\hline Reference & {$\left[f_{+}^{D \rightarrow \pi}\left(q^{2}=1 \mathrm{GeV}^{2}\right) / f_{D^{+}}\right] \mathrm{GeV}^{-1}$} \\
\hline ETMC [51] & $4.39 \pm 0.31_{\text {stat. }}$ \\
Becirevic et al (linear fit) [52] & $3.76 \pm 0.54$ \\
Becirevic et al (HMChPT fit) [52] & $4.32 \pm 0.56$ \\
CLEO [23, 54] & $4.51 \pm 0.53$ \\
\hline
\end{tabular}

Table 1: Results for $\left[f_{+}^{D \rightarrow \pi}\left(q^{2}=1 \mathrm{GeV}^{2}\right) / f_{D^{+}}\right] \mathrm{GeV}^{-1}$ from the $N_{f}=2$ calculations described in the text and CLEO experimental results.

The difference between the results coming from the two fits will be added as a source of systematic error.

The results from both works, together with the corresponding experimental number obtained from CLEO-c results in [23] and [54] (using the z-expansion parametrization) are in Table 1. Both analyses need to have a better control over the extrapolation to the physical masses. They also need to explicitly study discretization errors with simulations at several lattice spacings.

\section{4. $B^{0}-\bar{B}^{0}$ mixing}

The mixing in the $B_{q}^{0}-\bar{B}_{q}^{0}$ system is an interesting place to look for NP effects. The BSM effects can appear as new tree level contributions, or through the presence of new particles in the box diagrams. In fact, it has been recently claimed that there is a disagreement between the direct experimental measurement of the phase of $B_{s}^{0}$ mixing amplitude and the SM prediction [3]. Possible NP effects have also been reported to show up in the comparison between direct experimental measurements of $\sin (2 \beta)$ and SM predictions using $B^{0}$ mixing parameters [14]. Studies of neutral $B$ meson mixing parameters can also impose important constraints on different NP scenarios [55]. 
In the SM, the $B^{0}-\bar{B}^{0}$ mixing is due to box diagrams with exchange of two $W$-bosons. These box diagrams can be rewritten in terms of an effective Hamiltonian with four-fermion operators describing processes with $\Delta B=2$. The matrix elements of the operators between the neutral meson and antimeson encode the non-perturbative information on the mixing and can be calculated using lattice QCD techniques. Those matrix elements are parametrized by products of $B$ decay constants and bag parameters, which provide the value of quantities experimentally measurable, like the mass differences, $\Delta M_{s, d}$, and decay width differences, $\Delta \Gamma_{s, d}$, between the heavy and light $B_{s}^{0}$ and $B_{d}^{0}$ mass eigenstates. For example, the mass difference is given by

$$
\left.\Delta M_{s(d)}\right|_{\text {theor. }} \propto\left|V_{t s(d)}^{*} V_{t b}\right|^{2} f_{B_{s(d)}}^{2} \hat{B}_{B_{s(d)}},
$$

with $\left\langle\bar{B}_{s}^{0}\left|Q_{L}^{s(d)}\right| B_{s}^{0}\right\rangle=\frac{8}{3} M_{B_{s(d)}}^{2} f_{B_{s(d)}}^{2} B_{B_{s(d)}}(\mu)$ and $O_{L}^{s(d)}=\left[\bar{b}^{i} \gamma_{\mu}\left(1-\gamma_{5}\right) s^{i}\left(d^{i}\right)\right]\left[\bar{b}^{j} \gamma^{\mu}\left(1-\gamma_{5}\right) s^{j}\left(d^{j}\right)\right]$.

Many of the uncertainties that affect the theoretical calculation of the decay constants and bag parameters cancel totally or partially if one takes the ratio $\xi^{2}=f_{B_{s}}^{2} B_{B_{s}} / f_{B_{d}}^{2} B_{B_{d}}$. Hence, this ratio and therefore the combination of CKM matrix elements related to it, $\left|\frac{V_{t d}}{V_{t s}}\right|$, can be determined with a significantly smaller error than the individual matrix elements. The ratio $\xi$ is also an important ingredient in the unitarity triangle analyses and the search for BSM effects [14].

The first lattice calculation of the $B^{0}$ mixing parameters with $N_{f}=2+1$ sea quarks, which only studied the $B_{s}^{0}$ sector, was performed by the HPQCD collaboration in [56]. The authors obtained

$$
\Delta M_{s}=20.3(3.0)(0.8) p s^{-1} \quad \text { and } \quad \Delta \Gamma_{s}=0.10(3) p s^{-1},
$$

which is compatible with experiment.

The FNAL/MILC [57] and HPQCD [58] collaborations are currently working on a more complete study of $B^{0}-\bar{B}^{0}$ mixing, including $B_{s}^{0}$ and $B_{d}^{0}$ parameters. The main goal of both projects is obtaining the ratio $\xi$ fully incorporating vacuum polarization effects. The choice of actions and the setup is the same as for their $f_{B}, f_{B_{s}}$ calculations described in Section 2.2.

Figures 9 and 10 show some examples of the preliminary values of $f_{B_{q}} \sqrt{M_{B_{q}} B_{B_{q}}}$ obtained as function of light valence mass and light sea quark masses respectively. The renormalization of the matrix elements is done in both cases perturbatively at one-loop. However, the FNAL/MILC collaboration results for the matching coefficients are still preliminary, so only bare results from this collaboration are shown in Figure 9.

The dependency on the light sea quark mass is in both studies very mild as can be seen in the Figures, so only the chiral extrapolation in the $d$ quark mass for $B_{d}^{0}$ parameters is expected to be a significant source of error. Figure 10 also shows that the results for the two different lattice spacings are very similar, which indicates small discretization errors. The statistical errors in both cases and for parameters in both the $B_{s}^{0}$ and $B_{d}^{0}$ systems are in the range 1-4\%. Relativistic corrections after power law subtractions are under control in the HPQCD study. They are around 5-6\% for the coarse lattice and $3-4 \%$ for the fine lattice.

A comparison of the preliminary results from the two collaborations for the ratio $\xi$ is shown in Figure 11. Again, FNAL/MILC results are non-renormalized ones, but in the case of $\xi$ a strong cancellation of perturbative corrections is expected between numerator and denominator, so renormalized results are going to be shifted by less than a $1 \%$ with respect to the bare ones. This has been explicitly checked in the HPQCD analysis and in the FNAL/MILC analysis with the preliminary 


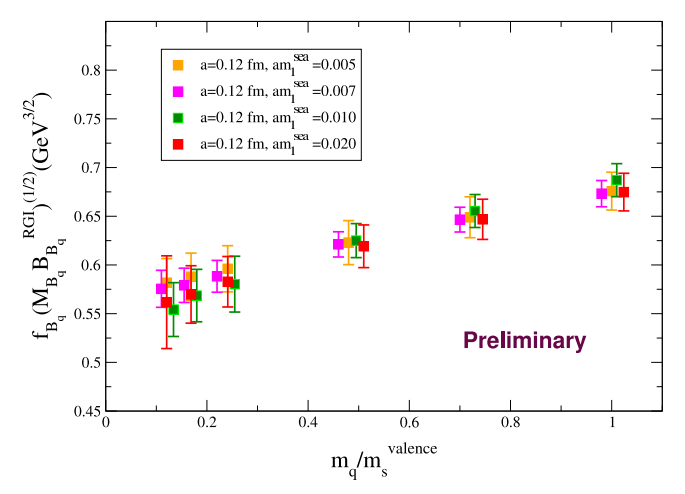

Figure 9: Bare values of $f_{B_{q}} \sqrt{M_{B_{q} B_{B_{q}}}}$ in lattice units as a function of the light valence quark mass $m_{q}$ normalized to the value of the physical strange quark mass from the FNAL/MILC collaboration. The results correspond to one of the three lattice spacings at which the FNAL/MILC's study is performed. The bottom valence quark is fixed to its physical value and the strange valence quark is very close to its physical value. The strange sea quark mass is also very close to its physical value.

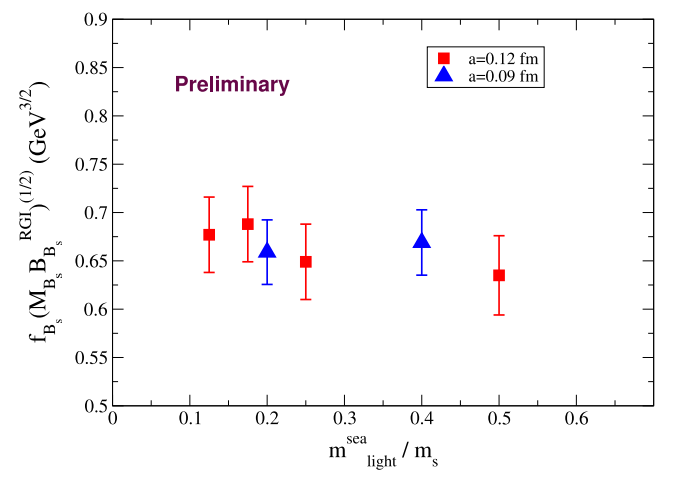

Figure 10: Values of $f_{B_{s}} \sqrt{M_{B_{s}} \hat{B}_{B_{s}}}$ in $\mathrm{GeV}^{3 / 2}$ as a function of the light sea quark mass normalized to the physical strange quark mass from the HPQCD collaboration. The data include statistical, perturbative and scale errors. The bottom valence quark is fixed to its physical value and the strange valence quark is very close to its physical value. The strange sea quark mass is also very close to its physical value.

matching coefficients. The differences between fine and coarse lattices in Figure 11 are small. This suggests small discretization effects. Another observation about that figure is that there is a very good agreement between the results from the two collaborations. Hence, heavy quark discretization effects, which are different for the two collaborations, must be small.

Figure 11 also contains the curve corresponding to the extrapolation to the physical $m_{d}$ after $m_{s}$ is fixed to its physical value and the continuum limit is taken, from the FNAL/MILC collaboration. That is done using SChPT at NLO plus analytic NNLO terms. The preliminary result from this collaboration after extrapolations are performed is $\xi=1.211 \pm 0.038 \pm 0.024$, where the second error is the sum in quadrature of the systematic uncertainties some of which are still under investigation.

The ALPHA collaboration has completed the computation of the non-perturbative renormalization and renormalization group running of the complete basis of four fermion operators with $\Delta B=2$ using $N_{f}=2$ dynamical Wilson fermions [60]. Heavy quarks are described in this analysis in the static limit. Using Wilson actions with suitable twisted mass terms, the mixing parameters can be related to matrix elements of parity-odd operators that are protected from extra mixings under renormalization due to the breaking of chiral symmetry. The precision of the final study of $B^{0}-\bar{B}^{0}$ parameters using these results is however limited due mainly to cut-off effects. That could be improved in the future by simulating at smaller values of the lattice spacing and/or improving operators at order $a$.

The RBC/UKQCD collaboration presented last year at this conference preliminary $N_{f}=2+1$ bare results for the $B^{0}-\bar{B}^{0}$ parameters [59]. This year the collaboration has discussed in this 


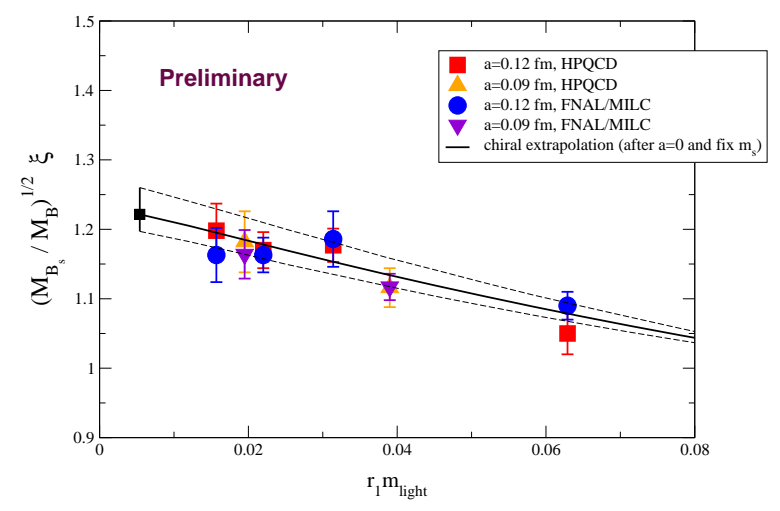

Figure 11: Product of the ratios $\xi$ and $M_{B_{s}} / M_{B_{d}}$ as a function of the down quark mass normalized to the strange quark mass. Results for both FNAL/MILC and HPQCD collaborations including only statistical errors are shown for two different values of the lattice spacing $a$. Only the full QCD points are shown for the FNAL/MILC collaboration.

conference the values of the renormalization coefficients calculated to one-loop [30]. The renormalization could partially correct the differences found in [59] between the results using different smearings.

\section{1 $B^{0}-\bar{B}^{0}$ mixing beyond the Standard Model}

The effects of heavy new particles in the box diagrams that describe the $B^{0}$ mixing can be seen in the form of effective operators built with SM degrees of freedom. The NP could modify the Wilson coefficients of the four-fermion operators that already contribute to $B^{0}$ mixing in the SM and gives rise to new four-fermion operators in the $\Delta B=2$ effective Hamiltonian -see [61, 62] for a list of the possible operators in the SUSY basis. The calculation of those Wilson coefficients for a particular BSM theory, together with the lattice calculation of the matrix elements of all the possible four-fermion operators in the SM and beyond and experimental measurements of $B^{0}$ mixing parameters, can constrain BSM parameters and help to understand NP.

To date, there does not exist an unquenched determination of the complete set of matrix elements of four-fermion operators in that general $\Delta B=2$ effective Hamiltonian. However, both FNAL/MILC and HPQCD collaborations are working on extending their analysis to BSM operators in the near future. Actually, the HPQCD collaboration has already calculated the one-loop matching coefficients needed for such an analysis [63].

\section{Heavy quark masses}

\subsection{Charm quark mass}

A new method to extract the charm quark mass has been proposed this year in [65]. The method is analogous to the extraction of $m_{c}$ from dispersion relations using perturbative determination of zero-momentum moments of current-current correlators and experimental data from 
$e^{+} e^{-} \rightarrow$ hadrons [66]. In [65], experimental data are substituted by lattice data. The methodology proposed in [65] is interesting in several ways. First of all, it provides a way of calculating $m_{c}$ that does not rely on lattice perturbation theory but continuum perturbation theory, for which higher orders in the expansion are known. The precision achieved is thus better than with the traditional methods. It also constitutes a way of checking the lattice discretization and techniques used in the calculation, since the result can be compared against the one coming from the pure continuum calculation. That would give confidence to apply the same discretization formalism and techniques to other charm quantities, like decay constants. Finally, the same methodology can be applied to different correlators to extract other masses, condensates, etc.

The charm quark mass is extracted in [65] from moments of charm quark correlators calculated with the HISQ action on $N_{f}=2+1$ MILC configurations. The moments are defined as $G_{n} \equiv$ $\sum_{t}(t / a)^{n} G(t)$, where the correlation function $G(t)$ is given by

$$
G(t) \equiv a^{6} \sum_{\vec{x}}\left(a m_{0 c}\right)^{2}\left\langle 0\left|j_{5}(\vec{x}, t) j_{5}(0,0)\right| 0\right\rangle,
$$

with $j_{5}=\bar{\psi}_{c} \gamma_{5} \psi_{c}$. On the other hand, those moments can be calculated in the continuum through the expression $G_{n}=\frac{g_{n}\left(\alpha_{\overline{M S}}(\mu), \mu / m_{c}\right)}{\left(a m_{c}^{\overline{M S}}(\mu)\right)^{n-4}}$, where $g_{n}\left(\alpha_{\overline{M S}}(\mu), \mu / m_{c}\right)$ is evaluated perturbatively and for some values of $n$ it is known to four loops. The normalization factors in (5.1) are such that the lattice and continuum expressions can be matched in the continuum limit, allowing the extraction of $m_{c}^{\overline{M S}}$ from that matching relation. Appropriate ratios of the moments, $R_{n}$, are defined so systematic uncertainties like the $O\left(\left(a m_{c}\right)^{n}\right)$ errors and the one associated with the tuning of $a m_{0 c}$ and the lattice spacing are suppressed. The resulting dependence on the lattice spacing is very mild, in particular for moments with $n \geq 8$, as can be seen in Figure 12. The authors in [65] tested the

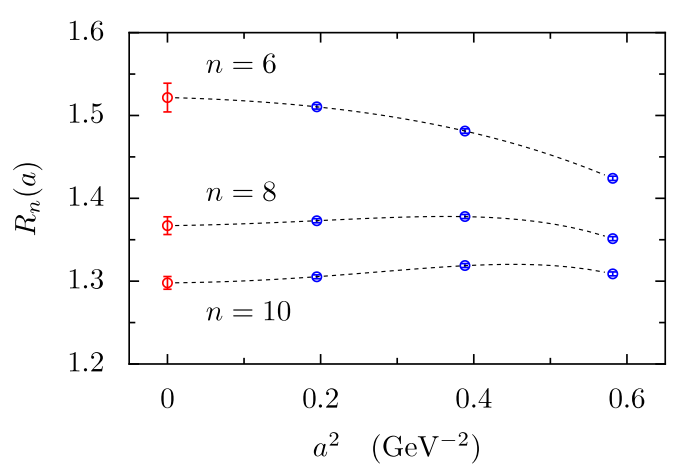

Figure 12: Extrapolation to the continuum for some of the moments $R_{n}$ analyzed to get the final value of $m_{c}$. Figure from [65].

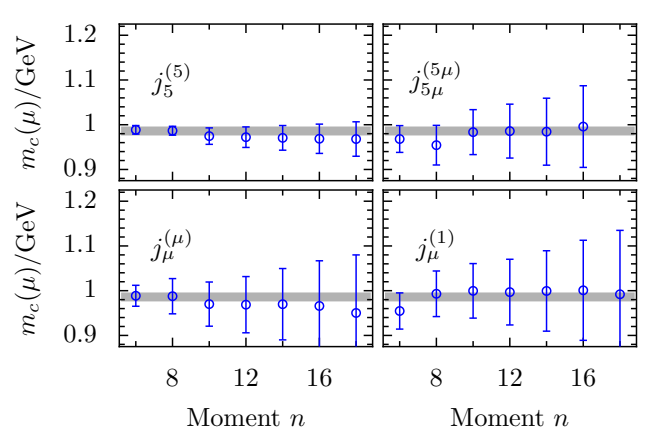

Figure 13: Values of $m_{c}(3 \mathrm{GeV})$ from different moments of correlators and different lattice operators. The grey band is the final result for the mass. Figure from [67].

impact of systematic errors and taste-changing effects due to the use of staggered fermions by calculating $m_{c}$ with different moments and different vector and axial correlators. Lattice artifacts enter in a different way in those calculations, so the agreement between them, shown in Figure 13, indicates that those artifacts are very much under control in this study.

Updated results from this analysis was presented at this conference [67] and later appeared in [65]. The updates include new simulations at a fourth, smaller, lattice spacing $(a=0.06 \mathrm{fm})$ and 
higher order terms in the continuum perturbative expansion for some of the moments. The updated value of the charm quark mass is $m_{c}^{\overline{M S}}(3 \mathrm{GeV})=0.986(10) \mathrm{GeV}$. This agrees perfectly with the one obtained from the purely continuum calculation using $e^{+} e^{-} \rightarrow$ hadrons data, $m_{c}^{\overline{M S}}(3 \mathrm{GeV})=$ $0.986(13) \mathrm{GeV}$.

The same methodology can be applied to the calculation of the bottom quark mass. This is being done by the same collaboration and preliminary results were also reported in [67]. They used the NRQCD action for the $b$ quarks. In this case, currents are not conserved and extra ratios of moments must be taken in order to cancel the renormalization factors. Their result is $m_{b}^{\overline{M S}}\left(m_{b}\right)=$ $4.20(4) \mathrm{GeV}$, that again agrees very well with the continuum one, $m_{b}^{\overline{M S}}\left(m_{b}\right)=4.16(3) \mathrm{GeV}$.

The HPQCD collaboration is also calculating $m_{c}$ with HISQ fermions on $N_{f}=2+1$ MILC configurations in a more standard way, using two-loop perturbation theory based on traditional methods and high- $\beta$ techniques. The preliminary value, $m_{c}^{\overline{M S}}(3 \mathrm{GeV})=0.983(25) \mathrm{GeV}$, [68] agrees with the one obtained from the current-current correlators method, but with significantly larger errors. The main goal of this calculation is obtaining an accurate determination of $m_{c} / m_{s}$ that, together with a precise value of $m_{c}$, will allow getting a value of $m_{s}$ with a very small error. It will be interesting to compare this value of $m_{s}$ with the one obtained in a direct way by the same collaboration with Asqtad fermions in [69].

Another on-going $N_{f}=2+1$ determination of $m_{c}$ and $m_{b}$ is the one by the FNAL/MILC collaboration, whose preliminary results were presented at this conference last year [70]. The main error there is the one associated with the truncation of the perturbative series.

\subsection{Bottom quark mass}

An accurate determination of the bottom quark mass is crucial for many continuum perturbation theory studies of heavy flavour observables. For example, the inclusive determination of $\left|V_{u b}\right|$ is very sensitive to the value of $m_{b}$ used in the theoretical analysis.

Besides the preliminary results presented for $m_{b}$ by the HPQCD collaboration using the currentcurrent correlators method and mentioned in the last section, only quenched determinations of this parameter have appeared recently.

The ALPHA collaboration calculated this parameter in the framework of HQET including $1 / m$ corrections in [71]. The result is $m_{b}^{\overline{M S}}\left(m_{b}\right)=4.347(48) \mathrm{GeV}$, very much compatible with the number quoted in [28], $m_{b}^{\overline{M S}}\left(m_{b}\right)=4.42(6) \mathrm{GeV}$. That second result is also obtained in the quenched approximation and in the framework of HQET going beyond the static limit, but it is based on the SS method. The setup is the same as that discussed for the $f_{B_{s}}$ in section 2.2. Again, it is important that these two different approaches agree, but an unquenched calculation is needed to have reliable results.

A completely different approach is followed by the TWQCD collaboration in [72]. That reference describes an exploratory study in the quenched approximation based on the use of relativistic domain wall fermions simulated in a very small volume (very small lattice spacing). They got $m_{c}^{\overline{M S}}\left(m_{c}\right)=1.16 \pm 0.04 \mathrm{GeV}$ and $m_{b}^{\overline{M S}}\left(m_{b}\right)=4.65 \pm 0.05 \mathrm{GeV}$, where the errors are estimates which do not include all the systematics. In [72], the authors predicted a mass of the $\eta_{b}$, $m_{\eta_{b}}=9383(4)(2) \mathrm{MeV}$ which agrees with the subsequent experimental measurement by the BaBar collaboration [73], $m_{\eta_{b}}=9388.9_{-2.3}^{+3.1} \pm 2.7 \mathrm{MeV}$. 


\section{Conclusions}

The non-perturbative input coming from lattice calculations of hadronic matrix elements in the heavy flavour sector is needed to extract Standard Model parameters and test the model itself by overconstraining the values of those parameters. The information encoded in such heavy flavour observables is complementary to direct searches in LHC to study and constrain New Physics effects. Hints of discrepancies between SM predictions and experimental measurements have started to show up in some CP violating observables $[64,14]$ in the $B^{0}-\bar{B}^{0}$ system and charm meson leptonic decays [12]. The precise determination of the CKM matrix elements $\left|V_{u b}\right|$ and $\left|V_{c b}\right|$, and the accurate calculation of parameters like $\xi$, decay constants, ..., involved in those analyses, is crucial in order to discern the origin of those disagreements and fully exploit the potential of the $\mathrm{CP}$ violating observables on constraining NP. Those calculations must be prioritized.

In order to be able to get the around 5\% accuracy required by phenomenology, vacuum polarization effects must be included in the simulations in a realistic way, i.e., $N_{f}=2+1$, and a rigorous study of systematic errors, including an analysis of the validity of the ChPT techniques used, must be performed. Such calculations are now possible. The use of techniques like double ratio methods, twisted boundary conditions and model independent parametrizations of the $q^{2}$ dependence of form factors have allowed (and/or will allow) to make important progress in achieving those goals.

New precise results have been presented at this conference for the $D$ and $B$ decay constants, the form factor describing $B$ semileptonic decays and the charm quark mass. Additional analyses of those parameters from other collaborations, together with final results for $B^{0}$ mixing parameters, $D$ semileptonic decay form factors, the bottom quark mass, will soon be available with errors at the few percent level. The extension of $B^{0}-\bar{B}^{0}$ studies in the SM to include BSM operators and to study short-distance contributions to $D^{0}-\bar{D}^{0}$ mixing in the SM and beyond are being pursued by the HPQCD and FNAL/MILC collaborations.

\section{Acknowledgments}

I thank Ian Allison, Benoit Blossier, Ting-Wai Chiu, Christine Davies, Aida El-Khadra, Todd Evans, Eduardo Follana, Benjamin Haas, Jack Laiho, Peter Lepage, Paul Mackenzie, Marco Panero, Silvano Simula, Junko Shigemitsu, Amarjit Soni, Nazario Tantalo, Cecilia Tarantino, Ruth Van de Water and Georg von Hippel for help in the preparation of this talk. I thank the organizers to invite me to this enjoyable meeting. This work was supported in part by the DOE under grant no. DE-FG02-91ER40677, by the Junta de Andalucía [P05-FQM-437 and P06-TIC-02302] and by the URA Visiting Scholars Program.

\section{References}

[1] T. Aaltonen et al. [CDF collaboration], Phys. Rev. Lett. 100 (2008) 121803 [arXiv:0712.2348 [hep-ex]].

[2] V. M. Abazov et al. [D0 Collaboration], arXiv:0802.2255 [hep-ex].

[3] M. Bona et al. [UTfit Collaboration], arXiv:0803.0659 [hep-ph].

[4] A. Abulencia et al. [CDF Collaboration], Phys. Rev. Lett. 97 (2006) 242003 [hep-ex/0609040]; 
[5] W. M. Yao et al. [Particle Data Group], J. Phys. G 33 (2006) 1.

[6] S. Stone, arXiv:0806.3921 [hep-ex].

[7] T. Skwarnicki, Proceedings of the Flavor Physics \& CP Violation (FPCP) conference, Taipei, Taiwan, May 5-9 (2008)

[8] B. Aubert et al. [BABAR Collaboration], Phys. Rev. Lett. 98 (2007) 091801 [arXiv:hep-ex/0612020].

[9] E. Barberio et al. [Heavy Flavor Averaging Group (HFAG) Collaboration], arXiv:0704.3575 [hep-ex].

[10] K. Jansen, PoS LAT2008 (2008) 010.

[11] L. Lellouch, PoS LAT2008 (2008) 015.

[12] B. A. Dobrescu and A. S. Kronfeld, Phys. Rev. Lett. 100 (2008) 241802 [arXiv:0803.0512 [hep-ph]]; PoS LAT2008 (2008) 282.

[13] G. von Hippel, R. Sommer, J. Heitger, S. Schaefer and N. Tantalo, PoS LATTICE2008 (2008) 227 [arXiv:0810.0214 [hep-lat]].

[14] E. Lunghi and A. Soni, Phys. Lett. B 666 (2008) 162 [arXiv:0803.4340 [hep-ph]]; PoS LAT2008 (2008) 281.

[15] C. Bernard et al. [Fermilab Lattice and MILC Collaborations], PoS LAT2007 (2007) 370; P. Mackenzie, C. Bernard and J. Simone, PoS LAT2008 (2008) 278.

[16] S. Naik, Nucl. Phys. B 316 (1989) 238; G. P. Lepage, Phys. Rev. D 59 (1999) 074502 [hep-lat/9809157]; K. Orginos, D. Toussaint and R. L. Sugar [MILC Collaboration], Phys. Rev. D 60 (1999) 054503 [hep-lat/9903032].

[17] A. X. El-Khadra, A. S. Kronfeld and P. B. Mackenzie, Phys. Rev. D 55 (1997) 3933 [arXiv:hep-lat/9604004].

[18] W. J. Lee and S. R. Sharpe, Phys. Rev. D 60 (1999) 114503 [arXiv:hep-lat/9905023]; C. Bernard [MILC Collaboration], Phys. Rev. D 65 (2002) 054031 [arXiv:hep-lat/0111051]; C. Aubin and C. Bernard, Phys. Rev. D 68 (2003) 034014 [arXiv:hep-lat/0304014]; ibid, 074011 [arXiv:hep-lat/0306026]; Nucl. Phys. Proc. Suppl. 140 (2005) 491 [arXiv:hep-lat/0409027].

[19] E. Follana, C. T. H. Davies, G. P. Lepage and J. Shigemitsu [HPQCD Collaboration], Phys. Rev. Lett. 100 (2008) 062002 [arXiv:0706.1726 [hep-lat]].

[20] E. Follana et al. [HPQCD Collaboration], Phys. Rev. D 75, 054502 (2007) [arXiv:hep-lat/0610092].

[21] C. Tarantino [European Twisted Mass Collaboration], PoS LATTICE2008 (2008) 285.

[22] R. Frezzotti and S. Sint, Nucl. Phys. Proc. Suppl. 106 (2002) 814 [arXiv:hep-lat/0110140].

[23] et al. [CLEO Collaboration], arXiv:0806.2112 [hep-ex].

[24] K. Abe et al. [Belle Collaboration], Phys. Rev. Lett. 100 (2008) 241801 [arXiv:0709.1340 [hep-ex]].

[25] A. Gray et al. [HPQCD Collaboration], Phys. Rev. Lett. 95 (2005) 212001 [arXiv:hep-lat/0507015].

[26] G. P. Lepage, L. Magnea, C. Nakhleh, U. Magnea and K. Hornbostel, Phys. Rev. D 46 (1992) 4052 [hep-lat/9205007].

[27] M. Della Morte, S. Durr, D. Guazzini, R. Sommer, J. Heitger and A. Juttner, JHEP 0802, 078 (2008) [arXiv:0710.2201 [hep-lat]].

[28] D. Guazzini, R. Sommer and N. Tantalo, JHEP 0801 (2008) 076 [arXiv:0710.2229 [hep-lat]]. 
[29] M. Guagnelli, F. Palombi, R. Petronzio and N. Tantalo, Phys. Lett. B 546 (2002) 237 [arXiv:hep-lat/0206023].

[30] T. Ishikawa, PoS LAT2008 (2008) 277.

[31] M. Wagner, PoS LAT2008 (2008) 122.

[32] E. B. Gregory, C. T. H. Davies, K. Y. Wong, E. Follana, E. Gamiz, G. P. Lepage and J. Shigemitsu, PoS LAT2008 (2008) 109 [arXiv:0810.1845 [hep-lat]].

[33] S. Hashimoto, A. X. El-Khadra, A. S. Kronfeld, P. B. Mackenzie, S. M. Ryan and J. N. Simone, Phys. Rev. D 61 (2000) 014502 [arXiv:hep-ph/9906376].

[34] P. F. Bedaque, Phys. Lett. B 593 (2004) 82 [arXiv:nucl-th/0402051]; C. T. Sachrajda and G. Villadoro, Phys. Lett. B 609 (2005) 73 [arXiv:hep-lat/0411033].

[35] S. Meinel, E. H. Muller, L. Khomskii, A. Hart, R. R. Horgan and M. Wingate, PoS LAT2008 (2008) 280 [arXiv:0810.0921 [hep-lat]].

[36] J. Laiho [Fermilab Lattice and MILC Collaborations], PoS LATTICE2007 (2007) 358 [arXiv:0710.1111 [hep-lat]].

[37] C. Bernard et al., arXiv:0808.2519 [hep-lat].

[38] S. Hashimoto, A. S. Kronfeld, P. B. Mackenzie, S. M. Ryan and J. N. Simone, Phys. Rev. D 66 (2002) 014503 [arXiv:hep-ph/0110253].

[39] G. M. de Divitiis, R. Petronzio and N. Tantalo, arXiv:0807.2944 [hep-lat].

[40] G. M. de Divitiis, R. Petronzio and N. Tantalo, JHEP 0710 (2007) 062 [arXiv:0707.0587 [hep-lat]].

[41] J. F. Kamenik and F. Mescia, Phys. Rev. D 78 (2008) 014003 [arXiv:0802.3790 [hep-ph]].

[42] R. S. Van de Water, PoS LAT2008 (2008) 279.

[43] R. S. Van de Water [Fermilab Lattice and MILC Collaborations], in preparation.

[44] M. C. Arnesen, B. Grinstein, I. Z. Rothstein and I. W. Stewart, Phys. Rev. Lett. 95 (2005) 071802 [arXiv:hep-ph/0504209]; T. Becher and R. J. Hill, Phys. Lett. B 633 (2006) 61 [arXiv:hep-ph/0509090].

[45] J. Charles et al. [CKMfitter Group], Eur. Phys. J. C 41 (2005) 1 [arXiv:hep-ph/0406184].

[46] C. Bourrely, I. Caprini and L. Lellouch, arXiv:0807.2722 [hep-ph].

[47] QCDSF collaboration, in preparation.

[48] D. Becirevic and A. B. Kaidalov, Phys. Lett. B 478 (2000) 417 [arXiv:hep-ph/9904490].

[49] R. Fleischer, S. Jager, D. Pirjol and J. Zupan, arXiv:0806.2900 [hep-ph].

[50] C. Aubin et al., Phys. Rev. Lett. 95 (2005) 122002 [arXiv:hep-lat/0506030].

[51] ETMC collaboration, in preparation.

[52] B. Haas, arXiv:0805.2392 [hep-lat].

[53] D. Becirevic, B. Haas and F. Mescia, PoS LAT2007 (2007) 355 [arXiv:0710.1741 [hep-lat]].

[54] D. Cronin-Hennessy et al. [CLEO Collaboration], Phys. Rev. Lett. 100 (2008) 251802 [arXiv:0712.0998 [hep-ex]]; S. Dobbs et al. [CLEO Collaboration], Phys. Rev. D 77 (2008) 112005 [arXiv:0712.1020 [hep-ex]]. 
[55] For a recent review see R.Fleischer; arXiv:0802.2882 [hep-ph].

[56] E.Dalgic et al.; Phys. Rev. D76, 011501(R) (2007).

[57] R. Todd Evans, E. Gámiz, A. X. El-Khadra and M. Di Pierro, PoS LAT2007 (2007) 354 [arXiv:0710.2880 [hep-lat]]; PoS LAT2008 (2008) 052

[58] E. Gámiz, C. T. H. Davies, G. P. Lepage, J. Shigemitsu and M. Wingate, PoS LATTICE2007 (2007) 349 [arXiv:0710.0646 [hep-lat]].

[59] C. Albertus et al. [RBC and UKQCD Collaborations], PoS LAT2007, 376 (2007).

[60] P. Dimopoulos, G. Herdoiza, F. Palombi, M. Papinutto, C. Pena, A. Vladikas and H. Wittig [ALPHA Collaboration], JHEP 0805 (2008) 065 [arXiv:0712.2429 [hep-lat]].

[61] F.Gabbiani et al.; Nucl.Phys.B 477, 321 (1996).

[62] D.Becirevic et al.; JHEP 0204:025 (2002).

[63] E. Gamiz, J. Shigemitsu and H. Trottier, Phys. Rev. D 77 (2008) 114505 [arXiv:0804.1557 [hep-lat]].

[64] A. J. Buras and D. Guadagnoli, Phys. Rev. D 78 (2008) 033005 [arXiv:0805.3887 [hep-ph]].

[65] I. Allison et al. [HPQCD Collaboration], arXiv:0805.2999 [hep-lat].

[66] J. H. Kuhn and M. Steinhauser, Nucl. Phys. B 619 (2001) 588 [Erratum-ibid. B 640 (2002) 415] [arXiv:hep-ph/0109084]; J. H. Kuhn, M. Steinhauser and C. Sturm, Nucl. Phys. B 778 (2007) 192 [arXiv:hep-ph/0702103].

[67] P. Lepage, PoS LAT2008 (2008) 224

[68] I. Allison, PoS LAT2008 (2008) 225

[69] Q. Mason, H. D. Trottier, R. Horgan, C. T. H. Davies and G. P. Lepage [HPQCD Collaboration], Phys. Rev. D 73 (2006) 114501 [arXiv:hep-ph/0511160].

[70] E. D. Freeland, A. S. Kronfeld, J. N. Simone and R. S. Van de Water [Fermilab Lattice Collaboration and MILC Collaboration], PoS LAT2007 (2007) 243 [arXiv:0710.4339 [hep-lat]].

[71] M. Della Morte, N. Garron, M. Papinutto and R. Sommer, JHEP 0701 (2007) 007 [arXiv:hep-ph/0609294].

[72] T. W. Chiu, T. H. Hsieh, C. H. Huang and K. Ogawa [TWQCD Collaboration], Phys. Lett. B 651 (2007) 171 [arXiv:0705.2797 [hep-lat]].

[73] B. Aubert et al. [BABAR Collaboration], Phys. Rev. Lett. 101 (2008) 071801 [arXiv:0807.1086 [hep-ex]]. 